\title{
Utility of Parametric Curves in Image Processing Applications
}

\author{
${ }^{1}$ Manish Dixit* and ${ }^{2}$ Sanjay Silakari \\ ${ }^{1}$ Madhav Institute of Technology \& Science, Gwalior, India, \\ ${ }^{2}$ University Institute of Technology, RGPV, Bhopal, India \\ dixitmits@gmail.com,ssilakari@yahoo.com
}

\begin{abstract}
Image Processing is dealing with images containing lots of points. By Interpolation of these points to form different curves are taken under consideration in this paper. The curve modification and fitting in image processing can use Bezier Curve applications in various ways and by modifying these curves better result are generated. Bezier curve and its applications have vast scope for research in image processing like curve fitting algorithm, pattern recognition and computer aided geometric design. Application of Bezier curve are widely implemented in face detection, smile detection, hand writing and digit recognition which give wide view of Bezier curves. In this paper we go through various concepts and its application which can be used in image processing for providing extra edge in this field.
\end{abstract}

Keywords: Parametric Curve, Parametric Surface, Space Curve, Bezier Curve, Interpolation, Curve Fitting, Reconstruction

\section{Introduction}

Curve fitting algorithms has many application areas in image processing, computer graphics, pattern recognition, and computer-aided geometric design. Cubic Bezier curves and B-splines curves are two of the most frequently used cubic curves. Bezier curve mainly focuses on fitting of image outlines. Curve fitting can be referred to as interpolation or approximation. The objective of the curve fitting algorithm is to use a minimum number of cubic curve pieces to approximate the image's outline with minimum distortion [1]. An image's outline cannot be fitted by a single cubic if it contains corners, Corners are points at which the outline's directions take a sharp turn contains more than two inflection points, has large curvature changes along certain portion of the curve. To overcome these problems piecewise approximation can be used. Basic application in which Bezier curve widely implemented are face detection, smile detection, emotion detection, handwriting and digit recognition etc. In graphics there are various ways to model various objects. A graphic system typically uses a set of primitives or geometric forms that are simple enough to be easily manipulated to represent or model a variety of objects .Geometric form that are often used as primitives include, in order of complexity: points, lines, polylines, polygons and polyhedral. More complex geometric forms include curves, curved surface patches and quadric surfaces. Forming of curve is an interactive process. Interpolating curve passes exactly through the data points. Control Points control the shape of the curve by pulling the part of the curve toward it and denoted as approximating curve.

\section{Curve Representation}

A curve can be described by sequence of coordinate data points that can be find out using analytic equation and is drawn using these generated points. Quality and accuracy of curves depend upon the represented points. Minimum points required to draw the curve 
are three(Except in case of line).It is not sufficient to draw a smooth representation of curve only using three points for that larger no of dense points are required.

Fig: Curve represented by points

\section{Parametric Curves}

Parametric representation of curves allows close and multivalued functions to be easily defined and replaces the slopes with target vectors. The parametric representation involves expressing relationship for the $\mathrm{a}$ and $\mathrm{b}$ not in terms of each other, but in the form of independent variables known as parameters. A single Parameter $\mathrm{u}$ is used to represent curves by expressing $a$ and $b$ in terms of two variables. A point can be represented as $\mathrm{p}(\mathrm{u})=[\mathrm{a}(\mathrm{u}) \quad \mathrm{b}(\mathrm{u})]^{\mathrm{T}}, \mathrm{u}_{\min } \leq \mathrm{u} \leq \mathrm{u}_{\max }$, this equation shows that coordinate of a point on the curve is a representation of its position vector. The value of $u$ is taken between 0 and 1 to normalize the parametric value.

\subsection{Bezier Curve}

Pierre Bezier gave the concept of a Bezier curve in the year 1970 while working in French Renault Car Company to make the use of curves and surfaces to design car body shapes [2]. The Bezier curve is a parametric curve which is defined by a minimum of three points consisting of an origin, endpoint and at least one control point. Unlike straight lines and circles where one of the variables can be set to discover a point on the line here in Bezier curve it may sample as many times as required from $t$ in $[0,1]$ to obtain that many points. Each of these points returned is a function of the control points on the origin and endpoint. The simplest Bezier curve is the straight line from one point P0 to another P1, with the parametric equation:

$$
B(t)=P_{0}+t\left(P_{1}-P_{0}\right)
$$

The Parametric form of quadratic Bezier curve is determined by three control points $\mathrm{P} 0, \mathrm{P} 1$, and $\mathrm{P} 2$.

$$
B(t)=(1-t)^{2} P_{0}+2 t(1-t) P_{1}+t^{2} P_{2}
$$

The Parametric form of a cubic Bezier curve is determined by four control points P0, P1, $\mathrm{P} 2$, and $\mathrm{P} 3$.

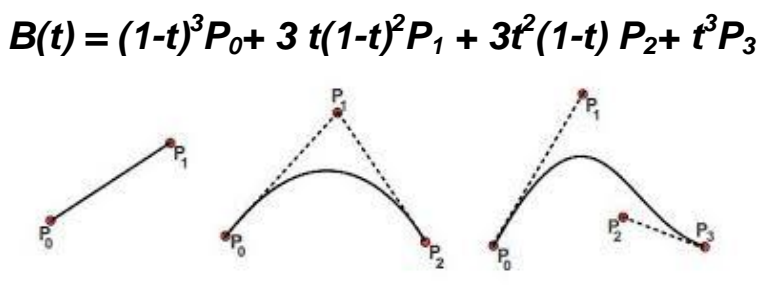

Figure 1. Straight Line, Quadratic and Cubic Bezier Curve

Overview Curve generation on constraining to pass exactly through the existing data points is a type of curve fitting [15]. This method is found to be robust and good when the basic shape is certainly determined by clear cut mathematical calculation like aircraft wings but where the aesthetic value and functionality is needed, combination of heuristic method and computational methods proves to be fruitful. Pierre Bezier invention Bezier curve meets both of these aspects [16]. The main feature of Bezier curve is the interplay between geometry and algebra that leads to a powerful theory of intuitive geometric construction. 


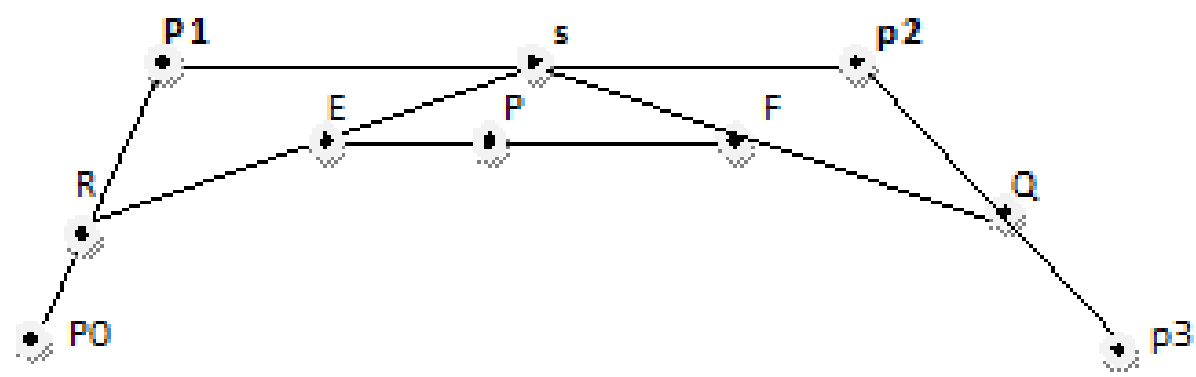

Figure 1. Bezier Curve Control Points

Bezier curves are based on four control points as shown in Figure 1, point $\mathrm{R}$ is placed between $P_{0}$ and $P_{1}$ at the given value of $u$ in fraction. Point $S$ and $Q$ are also placed between p1p2 and p2p3.E is placed at a fraction $u$ on $R$ to $S$ and $F$ between $S$ and $Q$. Finally, the desired point $\mathrm{P}$ is located at fraction $\mathrm{u}$ of the way from $\mathrm{E}$ to $\mathrm{F}$. The process is repeated for every $u$ between 0 and 1 , the curve $P(u)$ so generated from $P_{0}$, is attracted towards $P_{1}$ and $P_{2}$ and ends at $P_{3}$. This is how the Bezier Curve defined by four points. The Bezier curve can be generated by different configuration of four points. De Casteljau algorithm is generalizes gently for the case of $M+1$ control points $P_{1}, P_{2}, P_{3} \ldots P_{M}$. The general expression for a Bezier-Bernstein polynomial

$\mathrm{P}(\mathrm{u})=\sum_{\mathrm{K}=\mathrm{o}}^{\mathrm{M}} \frac{\mathrm{M} !}{(\mathrm{M}-\mathrm{K}) ! \mathrm{K} !} \mathrm{u}^{\mathrm{K}}(1-\mathrm{u})^{\mathrm{M}-\mathrm{K}^{\mathrm{K}} \mathrm{P}_{\mathrm{K}^{p}}} \quad 0<u \leq 1$

Here $0 !$ and $\mathrm{u}^{\mathrm{K}}=0$ when $\mathrm{u}$ and $\mathrm{k}$ are both 0 So

$\mathrm{P}(\mathrm{u})=\sum \mathrm{B}_{\mathrm{k}_{\mathrm{k}} \mathrm{M}}(\mathrm{u}) \mathrm{P}_{\mathrm{k}}$ or $\sum_{k=0}^{n} \mathrm{P}_{\mathrm{k}} \mathrm{B}_{\mathrm{k}}(\mathrm{u}), \quad 0 \leq \mathrm{u} \leq 1$

$\sum_{k=0}^{n} \mathrm{P}_{\mathrm{k}} \mathrm{B}_{\mathrm{k}}(\mathrm{u}), \quad 0 \leq \mathrm{u} \leq 1$

Where blending function $\mathrm{B}_{\mathrm{k}, \mathrm{M}}(\mathrm{u})$ is defined as

$\mathrm{B}_{\mathrm{k}, \mathrm{M}}(\mathrm{u})=\mathrm{C}\left(\mathrm{M}_{y} \mathrm{~K}\right), \mathrm{u}^{\mathrm{k}}(1-\mathrm{u})^{\mathrm{M}-\mathrm{K}}{ }_{y} \mathrm{C}\left(\mathrm{M}_{y} \mathrm{~K}\right)=\mathrm{M} ! /(\mathrm{M}-\mathrm{k}) ! \mathrm{k} !$
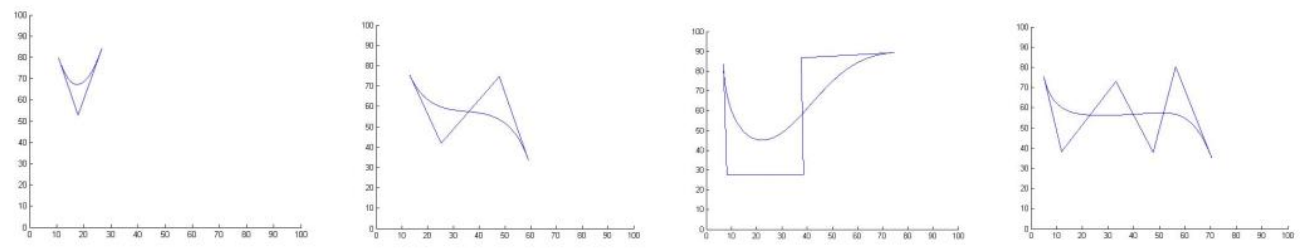

Figure 2. Sample of Bezier Curves with Different Control Points

\subsection{Properties}

There are lots of properties of Bezier curve which makes this curve strong to apply in image processing applications. The blending (basis) functions of Bezier curves are real and this curve has same end points as the guiding polygon which assures that curve always passes through the first and last control points. The degree of polynomial defining the segment of curve is always one less than the number of polygon points due to which the cubic Bezier curve lies within the convex hull formed by the four control points. The property of convex hull ensures that the polynomial for Bezier curve smoothly follows the 
control points. The direction of the tangent vector at the end points is the same as that of the vector determined by first and last segments. Bezier curve does not oscillate about any straight line more often than the defining polygon and invariant under an affine transformation.

\section{Review of Image Processing Applications of Bezier Curves}

There is lot of work done in the field of image processing using Bezier curves. Many image processing techniques uses this curve like image compression \& image segmentation. Various research work has been done using this curve like face recognition, facial expression detection, emotion extraction, smile detection, word recognition, character recognition, finger print recognition, human gait recognition and many more. The vast research is being done and various research papers are written on automatic affect analyze and recognition of human emotion [2]. Emotion recognition via facial expression is given by Ekam [4] which was based on Psychology. Ira et al. [5] gives architecture of hidden markov models which automatically segment and detect human facial expression from video sequences. Bertalmio et al. [6] first gives the method for computational impainting that is based on partial differential equation (PDE). Some more methods were also given based on the idea of Bertalmio et al. Drori et al. [7] gives the Fragment based image completion. He gives the method in which iteratively approximate the unknown parts and select adaptive image fragment to restore the image. Confidence map is used to restore the image. Its result was good but the speed was slow. Criminisi et al. [8] proposed exemplar - based image impainting algorithm which preserve the linear structure by reordering the synthesizing process based on their priority.A work is done in the field of Finger print data compression using Bezier curve. Various approaches are given for compression of finger print. AwadKh. Al-Asmari [9] gives the finger print image compression method via edge detection .In this method image is decomposed into two component, the component which encloses the edge is the first component called primary component, the part which contain the textures and features is the another component called secondary component. S. S. Gornale et al. [10] uses different transforms of wavelet packet and gives compression ratio for noisy and noiseless finger print images. In their research they show that by taking appropriate threshold value compression ratio can be increased. Song Zhao and Xiao-Fei Wang [11] gives compression algorithm, Wavelet-Based counterlet Transform (WBCT) for finger print images. He works on wavelet transform and directional filter banks that can be used to approximate natural images containing contours and oscillatory pattern. A method based on maximally-flat filter which implements the directional filter banks was used to minimize the frequency scrambling. A quad tree method was used for sorting which is similar to SPIHT was used to form WBCT coefficient classes explicitly. These classes were encoded by arithmetic and trellis-coded quantization scheme. There is constant improvement in present encoded algorithm over the SPIHT method and present method preserves fingerprint image details finely. S. Esakkirajan et al. [12] used counterlet transform and multistage vector quantization to compress the finger prints image. By their work it was shown that multistage vector quantization was good for low bit rate image and peak signal to noise ratio by wavelet transform is lower than counterlet transform. He also shows that by using counterlet transform, image reconstruction is achievable with less number of bits. In their work they showed that system gives better compressed image around 0.5 bits per dimension and very good results around 1 bits per dimension. Gulzar A. Khuwaja [13] works on data compression scheme with the best parameter for fingerprint images. His work mainly focuses to reduce the transmission cost together with maintain the person's identity. He chooses the wavelet packet filters, decomposition level and sub-bands which are better adapted with the frequency characteristics. If lower entropy or minimum distortion is consider then one may achieve better results i.e., better 
image representation .Image quality parameters like mean square error (MSE) and peak signal to noise ratio (PSNR) are used to figure out the performance of different wavelet filters. Work has also done in the field of digit recognition using Bezier Curve. S. Mahmoud [14] proposed system for the recognition of hand written Indian numerals. He uses a set of 120 features figure over angle span, distance span, horizontal span and vertical span for this. The HMM and 1-NN were used as classifiers and different sets of 120 features used to test the classifiers and observed that HMM classifier gives better result than 1-NN. D. Sharma et al. [15] gives method name Zone feature extraction for hand written numeral recognition. This method divides the image into $4 \times 4$ zones. Next to get more accurate results zones are further divided into $6 \times 6$ zones and this division is carried up to $8 \times 8$. Densities of object pixel are taken as features. He uses 1-NN classifier for classification and recognition. S. Impedove et al. [16] proposed a prototype generation technique in which he uses binary histogram for feature extraction of oriented gradients and $\mathrm{k}-\mathrm{NN}$ classifiers is used to decrease the classification time. The first step in this process was to determine the number of prototypes .Adaptive resonance theory concept was used to pick out the initial solution, and to get the final output an evolution strategy was used as second step. Aissa Kerkour El Miad and Azzeddine Mazzroui [17] proposed an approach to recognize Arabic numerals. The performance of the system for numeral recognition depends upon choice of features and classification system used. The characteristic dots of the Arabic digit whose shapes are closer to their associated Bezier curve. The k-nearest classifier is used in this Arabic numeral recognition system. The Gait Recognition is another application of image processing using Bezier curve. Lot of research work is done in this field. Yanmei et al. [18] gives the method for gait recognition. In their work they focuses on dynamic vision sensor(DVS) of the pixel position for gait sequence and then construct a dynamic variance matrix, by calculating their variance feature for gait identification. Wang and Liu [19] proposed the method on positioning body joints. Based on geometrical view while walking, firstly the coordinates of joints are calculated and then based on joints coordinates limbs angle are calculated and then Discrete Fourier transform is made. The amplitude and phase frequency of angles is selected as gait feature and finally objects are classified using nearest neighbor classifier. Zarina [20] works for recognition of human emotion from still images. In her two step work original image is used to analyze the facial expression and then facial emotion of features are verify in the region of interest. Pratibha mishra [21] gives the algorithm for gait recognition. She has considered the side view only, as the side view provides a lot of information of an individual walking. In her work firstly Bezier curve is generated based on an individual walk and recognition is performed by computing mean and variance and then matching those curves. The work done for raindrop detection [2226] till now supposes a sphere section for modeling droplet boundary. Gravity causes a unidirectional droplet deformation on the tilted planes but the assumption is not correct. Kurihata et al. [23] works on machine learning approach by template matching detects raindrops on the windshield from in-vehicle camera images. When the raindrop is in the sky results were good but for non -sky regions results were not as good. Zhang et al. [33] detects the blur image with motion analysis using wavelet transform and recognize optical contamination using cumulative differences. Their algorithm is good for rigid and opaque contamination but fails for raindrops as their look depends on background. Yamashita et al. remove the restrictions of hardware such as multiple camera [27-28] or pan-tilt surveillance camera [29-30] with known yaw rates to bypass the challenge of modeling. 


\section{Analysis and Result of Applications of Bezier curve in Image Processing}

\subsection{Detection of Skin Color}

One of the applications of Bezier Curve is detection of skin color using $\mathrm{YCbCr}$. For skin color segmentation, first we contrast the image. Then skin color segmentation is done. Then, we have to find the largest connected region. Then we have to check the probability to become a face of the largest connected region. If the largest connected region has the probability to become a face, then it will open a new form with the largest connected region. If the largest connected regions height $\&$ width is larger or equal than 50 and the ratio of height/width is between 1 to 2 , then it may be face.

\subsection{Face Recognition}

FACE identification and recognition is a biometric method used to identify the person's identity for security issues and surveillance. There are various facial recognition algorithm works on various features of face like size, shape of the eyes, nose, mouth, lips ,cheek bones, facial boundary, chin shape and jaw. Face recognition can be done using interpolated and approximated parametric Bezier and Spline Curves. The major facial feature areas are segmented, feature points are extracted and principal curves are obtained .On the basis of various distance measures curves are compared and identify the faces.

\subsection{Emotion Detection}

There are various types of emotion an individual's has like smile, disgust, sadness, happiness, angry and many more. For emotion detection of an image, finding the curves of the various facial features like lips, left eye , right eye, mouth, stretched features of cheeks are required than compare these curves with curves of similar normal emotion image. Comparing these digital curves on the basis of distance measures and shape information expression are recognized. Curves formed on Width and height of various features is another way to find out different emotions. Bezier curve, B-Spline curve and other parametric curves are used for this purpose. If the person's emotion information is available in the database, then the program will match which emotion's height is nearest the current height and the program will give the nearest emotion as output.

\subsection{Handwriting Detection}

The recognition of handwriting text is a topic widely studied by the scientific community using the digital curves. Some studies are devoted to the digit recognition given its growing interest in many applications such as postal mail sorting and bank check processing. The performances of recognition systems depend strongly on the choice of approaches used in the feature extraction approach and the classification techniques relating to training and testing phases. One of the methods is by using Bezier Curve that allows representing parametric curves from a limited number of data (some characteristic dots with their derivatives). Indeed, the characteristic dots of the Arabic digit that adopted are, those such that their associated Bezier curve is close to the shape of the digit which uses the k-nearest classifier.

\subsection{Fingerprint Recognition}

There are many application areas where fingerprints are to be recognized in biometric verification and to find out the culprit in many areas. One good example of biometric application is the forensic where we find out the culprit by their finger print matching. So 
there is vast amount of data in form of fingerprint data and to manage this data different approaches were taken and one of them is the image compression technique. Using shape information of ridges in the form of parametric equation of curve and its comparison finger print are verified.

\subsection{Raindrop Detection}

Recognize the shape of raindrop in the sky and after falling on the object. The shape of the raindrop depends on many factors like gravity etc. Lot of work done for the recognition of raindrop shape using the Bezier curves.

\subsection{Gait Recognition}

Human Gait recognition is a biometric technique to verify the identity of a person and also gender information. Gait means a manner of walking, running or stepping. So, to identify an individual by the way he walk is Gait recognition. It is not only limited to security applications but also use in medical field like to recognize Parkinson's disease and multiple sclerosis in their earliest stages. Using the human gait recognition even the maturity level of mind and the nature of its working at that instant is judged.

\subsection{Image Impainting}

Image impainting is a method to restore the image which is old or to remove the unwanted part from image. In this technique the lost and broken part of the image filled automatically. There is a lot of research work in this field of image impainting.

\section{Conclusion}

In this paper the uses, application and significance of digital and parametric curves are emphasized specifically the Bezier curve. By of different researches on the Bezier curve and its application we get so many approaches under consideration different curve by interpolating the control points in image processing.

\section{References}

[1] Lejun Shao and hao zhou," Curve Fitting with Bezier Cubics", School of EEE, Nanyang Technology University, Nanyang Avenue, Singapore 2264, Singapore, 1996, Vol. 58,

[2] Bezier curve, Wikipedia, 2013, http://en.wikipedia.org/wiki/Bezier_curve

[3] G. H. and P. M., "Automatic Temporal Segment Detection and Affect Recognition from Face and Body Display", IEEE Transactions on Systems, Man, and Cybernetics - Part B, vol. 39, no. 1, (2009), pp. 64-84.

[4] P. Ekman and W. Friesen, "Facial Action Coding System (FACS): Investigator's Guide. Conculting Psychologists Press, (1978).

[5] I. Cohen, A. Garg, and T. S. Huang, "Emotion Recognition from Facial Expressions using Multilevel HMM", In Proc. of the 2000 NIPS Workshop on Affective Computing, Denver, Colorado, USA, (2000) December.

[6] M. Bertalmio, G. Sapiro, V. Caselles, and C. Ballester, "Image inpainting," in Proceeding ACM Conference Computer Graphics, (2000), pp. 417-424.

[7] I. Drori, D. Cohen-Or, and H. Yeshurun, "Fragment based image completion", ACM Transactions on Graphics, vol. 22, (2003), pp. 303-312.

[8] A. Criminisi, P. P'erez, and K. Toyama, "Region filling and object removal by exemplar-based image inpainting," IEEE Transactions on Image Processing, vol. 13, (2004), pp. 1200-1212.

[9] Awad Kh. Al-Asmari, "Progressive Fingerprint Images Compression Using Edge Detection Technique", International Journal of Images Systems \& Technology, John Wiley \& Sons, vol. 12, (2002), pp. 211 216.

[10] S. S. Gornale, Vikas T Humbe, R. R. Manza and K. V. Kale, "Fingerprint Image Compression using Retain Energy (RE) and Number of Zeros (NZ) through Wavelet Packet (WP)", International Journal of Computer Science and Security, vol. 1, no. 2, (2008), pp. 35-42.

[11] S. Zhao and X.-F. Wang, "Fingerprint Image Compression Based on Directional Filter Banks and TCQ", 2009 Second International Workshop on Knowledge Discovery and Data Mining, pp. 660-663, 
[12] S. Esakkirajan, [21] used counterlet transform and multistage vector quantization to compress the finger prints image.

[13] G. A. Khuwaja [35] works on data compression scheme with the best parameter for fingerprint images.

[14] S. Mahmoud, "Recognition of writer-independent off-line handwritten Arabic (Indian) numerals using hidden Markov models", Signal Processing, vol. 88, no. 4, (2008), pp. 844-857.

[15] D. Sharma, and D. Gupta, "Isolated Handwritten Digit Recognition using Adaptive Unsupervised Incremental Learning Technique", International Journal of Computer Applications, vol. 7, no. 4, (2010), pp. 27-33, 2009.

[16] S. Impedovo, F. M. Mangini and D. Barbuzzi, "A novel prototype generation technique for handwriting digit recognition", Pattern Recognition, Available online 3 May (2013), http://dx.doi.org/10.1016/j.patcog.2013.04.016

[17] A. K. E. Miad and A. Mazzroui [15] proposed an approach to recognize Arabic numerals. In his method he used Hermite data from the digit shape.

[18] Y. C. J. Ren, R. Zhao and J. Jia, "Automatic Gait Recognition using Dynamic Variance Features", IEEE (2006).

[19] A.-H. Wang, J.-W. Liu, “A gait recognition method based on positioning human body joints”, IEEE (2007).

[20] Zareena, "Human Emotion Detection and Recognition from Still Images" Int. J. Computer Technology \& Applications (IJCTA), vol. 5, (2014).

[21] P. Mishra, "Human Gait Recognition using Bezier curve" / International Journal on Computer Science and Engineering (IJCSE), vol. 3, (2011).

[22] J. Halimeh and M. Roser, "Raindrop detection on car windshields using geometric photometric environment construction and intensity-based correlation", In IEEE Intelligent Vehicle Symposium (IV '09), Xi'an, China, (2009).

[23] H. Kurihata, T. Takahashi, I. Ide, Y. Mekade, H. Muraseand Y. Tamatsu, and T. Miyahara, "Rainy weather recognition from in-vehicle camera images for driver assistance", In IEEE Intelligent Vehicles Symposium (IV '05), (2005), pp. 205-210.

[24] D. Marquardt, "An algorithm for least-squares estimation of nonlinear parameters", SIAM Journal of Applied Mathematics, vol. 11, (1963), pp. 431-441.

[25] S.G. Narasimhan and S. K. Nayar, "Vision and the atmosphere. International Journal of Computer Vision, vol. 48, no. 3, (2002), pp. 233-254.

[26] M. Roser and A. Geiger, "Video-based raindrop detection for improved image registration", In IEEE Workshop on Video-Oriented Object and Event Classification (in conjunction with ICCV '09), )(2009).

[27] Y. Tanaka, A. Yamashita, T. Kaneko, and K. T. Miura, "Removal of adherent waterdrops from images acquired with a stereo camera system”, IEICE - Transactions on Information Systems, (2006).

[28] P. J. G. Teunissen. Adjustment theory: an introduction. Delft University Press, Postbus 98, 2600 MG Delft, (2000).

[29] N. Vasconcelos and A. Lippman, "Empirical bayesian em-based motion segmentation", In Proc. of the IEEE Conf. on Computer Vision and Pattern Recognition, Puerto Rico, (1997), pp. 527-532.

[30] A. Yamashita, I. Fukuchi, T. Kaneko, and K.T. Miura. Removal of adherent noises from image sequences by spatio-temporal image processing. IEEE International Conference on Robotics and Automation (ICRA '08), (2008), pp. 2386-2391.

[31] A. Yamashita, T. Harada, T. Kaneko, and K. T. Miura, "Removal of adherent noises from images of dynamic scenes by using a pan-tilt camera. IEEE/RSJ International Conference on Intelligent Robots and Systems (IROS '04), vol. 1, (2004).

[32] A. Yamashita, M. Kuramoto, T. Kaneko, and K. T. Miura, “A virtual wiper - restoration of deteriorated images by using multiple cameras. IEEE/RSJ International Conference on Intelligent Robots and Systems (IROS '03) vol. 3, (2003).

[33] Y. Zhang, J. Yang, K. Liu, and X. Zhang, "Self-detection of optical contamination or occlusion in vehicle vision systems", Journal of Optical Engineering, vol. 47, no. 6, 067006, (2008).

\section{Authors}

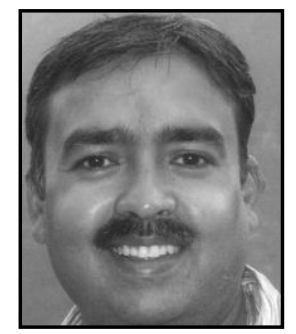

Manish Dixit, he received the B.E.in Computer Technology from Barkatullah University, Bhopal and M. E. in Communication Control and Networking from MITS, Gwalior In 1993 and 2006 respectively. He is pursuing his $\mathrm{PhD}$ from Rajiv Gandhi Technical University, Bhopal. He is currently working as Reader in the Department of Computer Science and Information Technology, MITS, Gwalior, India. He has presented various research papers in 
National and International Conferences and Journals. He is a Member of CSI, IETE, IEEE.

Sanjay Silakari, he is the Dean in faculty of CS/IT and the chairman of board of studies, CSE, RGPV, Bhopal. He is also a professor and Head of Department of CSE in University Institute of Technology of RGPV. He has more than two decades of teaching and administrative experience and has guided several students for their doctoral and master studies. He has several research publications to his credit in different reputed national and internal conferences and journals. He is a life member of ISTE, CSI, IAENG and a member of IEEE and ACM. 
International Journal of Signal Processing, Image Processing and Pattern Recognition Vol.8, No.7 (2015) 